\title{
The On-going Challenge of Pediatric Septic Shock: Optimizing Early Identification and Accurate Resuscitation
}

\author{
Jay D Fisher*
}

Department of Emergency Medicine, University Medical Centre, University of Nevada School of Medicine, Las Vegas, USA

*Corresponding author: Jay D Fisher, Department of Emergency Medicine, University Medical Center, University of Nevada School of Medicine, Las Vegas, USA, E-mail: jfisher@usacs.com

Received date: January 27, 2018; Accepted date: January 30, 2018; Published date: February 07, 2018

Citation: Fisher JD (2018) The On-going Challenge of Pediatric Septic Shock: Optimizing Early Identification and Accurate Resuscitation. J Intensive Crit Care. Vol.4 No.1:6

\section{Commentary}

Shock in the pediatric emergency department is frequently a diagnostic and therapeutic challenge. The child arrives in an undifferentiated manner and there can be dramatic clinical overlap between a variety of pathophysiologic states, caused by conditions ranging from immediately life-threatening to relatively innocuous. As a member of a small group of young pediatric emergency physicians in the 1990's, our group initiated an observational study to examine our performance utilizing standard pediatric advanced life support measures [1]. We strove to identify patients early and initiate rapid but measured fluid replacement, as well as clinically indicated vasopressor administration and antibiotics. Our patients received an average of $58 \mathrm{ml} / \mathrm{kg}$ of fluid over the course of a 4-5 h ED course. During the 8 year period of data collection, septic shock represented $57 \%$ of our cases. Hypovolemic shock, distributive and cardiogenic shock contributed $24 \%, 14 \%$ and $5 \%$ respectively. Our mortality was $6 \%$ overall and $5 \%$ in patients with septic shock and an identified pathogen.

As we accumulated our data, early goal directed therapy and the surviving sepsis campaign began, and with it came an even more aggressive fluid resuscitation strategy. Children were to be treated with $60 \mathrm{ml} / \mathrm{kg}$ of crystalloid over the course of 30 to 60 $\min$ [2]. Given that a child's intravascular volume is $80 \mathrm{ml} / \mathrm{kg}$, our group was hesitant with these recommendations-we had seen first-hand the potential complications of this therapy, specifically acute respiratory failure in the ED requiring emergent intubation and mechanical ventilation. One such patient enrolled late in the study period, after the Surviving Sepsis campaign had been widely disseminated and impacted our practice, is vividly recalled. This was a two month old female presented with fever for three day. The child demonstrated tachycardia, but was awake and alert with normal oxygen saturation. Pyelonephritis was quickly diagnosed. Her pulses were initially palpable, but after an initial $20 \mathrm{ml} / \mathrm{kg}$ bolus of saline and ceftriaxone administration, her extremities were cold, mottled and she had pulses that were barely palpable. Was this just transient endotoxin release that would resolve with time or did it represent dissemination of infection and worsening capillary leak? Following peripheral pulses, breath sounds and liver edge as a guide to therapy, $40 \mathrm{ml}$ per $\mathrm{kg}$ of saline was rapidly administered. The patient developed grunting, laboured respirations and increasing oxygen requirement. The decision to initiate mechanical ventilation was made. Laryngoscopy was difficult, and the patient's decreased residual capacity and alveolar capillary leak lead to rapid oxygen desaturation. The patient was successfully intubated on the third attempt with transient bradycardia but without the need for chest compressions. The child remained intubated for a week but survived. Did we overshoot this child's resuscitative requirements, or was the respiratory failure and difficult intubation inevitable?

Because of cases like this one, the initial enthusiasm for a new therapeutic strategy (early goal directed therapy) has come under further scrutiny [3]. Rapid administration of saline bolus volumes that approximate the patient's entire intravascular volume has not been uniformly successful and mortality in septic shock has not been dramatically reduced [4]. A recent meta-analysis on the association of fluid balance and outcomes in critically ill children demonstrated a 6 percent increase in the odds of mortality for every $1 \%$ increase in fluid overload [5]. Perhaps the measuring tools at our disposal for assessing the clinical impact of fluid therapy, such as clinical assessment of peripheral perfusion or ultrasonographic assessment of the inferior vena cava, are too blunt to predict the impact of aggressive fluid resuscitation at the cellular and mitochondrial level.

The bright news is that the wheels of medical progress continue to churn, and it is undeniable that the research on pediatric septic shock over the last twenty years has led to greater focus on this clinical challenge, earlier identification of patients, reduced variation in therapy and a move forward. An on-going study (Squeeze Trial) comparing early goal directed therapy with a fluid sparing approach in pediatric septic shock is on-going [6]. In the interim, clinical pathways leading to early identification of patients, quick administration of broadspectrum antibiotics, and aggressive-but careful-fluid resuscitation, will lead to optimal outcomes.

\section{References}

1. Fisher JD, Nelson DG, Beyersdorf H, Satkowiak LJ (2010) Clinical spectrum of shock in the pediatric emergency department. Pediatr Emerg Care 26: 622-625. 
2. Brierly J, Carcillo JA, Choong K, Cornell T, Decaen A, et al (2009) Clinical practice parameters for hemodynamic support of pediatric and neonatal septic shock: 2007 update from the American college of critical care medicine. Crit Care Med 37: 666-688.

3. Abulebda K, Cvijanovich NZ, Thomas NJ, Allen GL, Anas N, et al (2014) Post-ICU admission fluid balance and pediatric septic shock outcomes: A risk-stratified analysis. Crit Care Med 42: 397-403.

4. Larsen GY, Mecham N, Greenberg R (2011) An emergency department septic shock care guideline for children initiated at triage. Pediatrics 127: e1585-1592.
5. Alobaidi R, Morgan C, Basu RK, Stenson E, Featherstone R, et al (2018) Association between fluid balance and outcomes in critically ill children. A systematic review and meta-analyis. JAMA Pediatr 4540.

6. Parker $\mathrm{Mj}$, Thabane L, Fox-Robichaud $\mathrm{A}$, Liaw $\mathrm{P}$, Choong $\mathrm{K}$, et al (2016) A trial to determine whether septic shock-reversal is quicker in pediatric patients randomized to an early goal-directed fluid-sparing strategy versus usual care (SQUEEZE): Study protocol for a pilot randomized controlled trial. Trials 17: 556. 